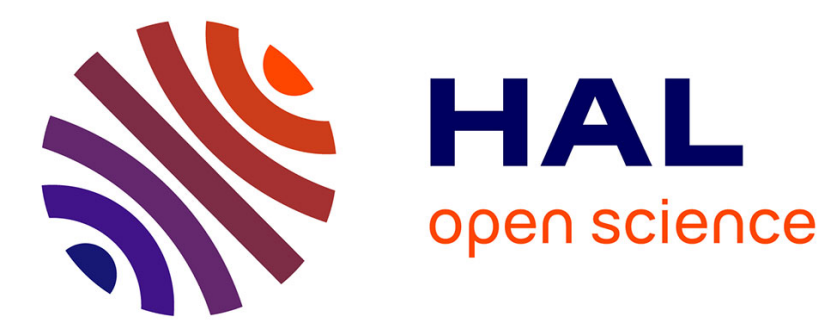

\title{
Flow energy harvesting with piezoelectric flags
}

\author{
Olivier Doaré, Sébastien Michelin, Miguel Pineirua, Yifan Xia
}

\section{To cite this version:}

Olivier Doaré, Sébastien Michelin, Miguel Pineirua, Yifan Xia. Flow energy harvesting with piezoelectric flags. 33rd International Conference on Ocean, Offshore and Arctic Engineering, ASME, Jun 2014, San Francisco, United States. 10.1115/OMAE2014-23867 . hal-01135610

\section{HAL Id: hal-01135610 https://hal.science/hal-01135610}

Submitted on 27 Mar 2015

HAL is a multi-disciplinary open access archive for the deposit and dissemination of scientific research documents, whether they are published or not. The documents may come from teaching and research institutions in France or abroad, or from public or private research centers.
L'archive ouverte pluridisciplinaire HAL, est destinée au dépôt et à la diffusion de documents scientifiques de niveau recherche, publiés ou non, émanant des établissements d'enseignement et de recherche français ou étrangers, des laboratoires publics ou privés. 


\title{
FLOW ENERGY HARVESTING WITH PIEZOELECTRIC FLAGS
}

\author{
Olivier Doaré \\ UME, ENSTA-Paristech, Palaiseau, France \\ Email: olivier.doare@ensta-paristech.fr
}

Sébastien Michelin
LadHyX, École Polytechnique, Palaiseau, France
Email: sebastien.michelin@ladhyx.polytechnique.fr

Miguel Pineirua

UME, ENSTA-Paristech, Palaiseau, France

Email: miguel.pineirua@ensta-paristech.fr

\author{
Yifan Xia \\ LadHyX, École Polytechnique, Palaiseau, France \\ Email: yifan.xia@ladhyx.polytechnique.fr
}

\begin{abstract}
In this article, energy harvesting with a fluttering cantilevered plate covered by piezoelectric patches in an axial flow is adressed. A theoretical model is presented which is then discretized and numerically integrated to perform a parametric study of the energy harvesting efficiency of the system. When one, two or three piezoelectric patches cover the plate, the optimal distributions of the patches that maximize the efficiency are obtained. Experimental results are presented, which are in good agreement with the model. When a significantly high number of patches of small size are considered, a continuous model is used to study the influence of a resonant harvesting circuit. A lock-in phenomenon is evidenced, which is able to significantly increase the efficiency.
\end{abstract}

Published in the proceedings of 33rd International Conference on Ocean, Offshore and Arctic Engineering OMAE2014, June 8-13, 2014, San Fransisco, USA Paper no OMAE2014-23867

\section{I - INTRODUCTION}

Recent efforts in the developement of new energy harvesting methods have been focused on the study of fluid-solid coupled systems that allow the conversion of the kinetic energy of flows into electrical energy [1, 2]. Apart from the classical technologies such as wind and water turbines, other techniques involving the deformation of elastic structures under the action of fluid forces have attracted the attention of several research groups. The present study is focused on the energy harvesting from the fluttering of a piezoelectric flag in an axial flow [3, 4].

A flexible plate in an axial flow can become unstable and develop self sustained flapping if the flow velocity exceeds a critical value. If the flapping plate is made of a piezoeletric material, the plate's deformation can be transformed into electrical energy. Previous works $[5,6]$ have studied the fully coupled dynamics of a flexible plate covered by an infinite number of piezoelectric patches connected to simple resistive circuits. The case of a unique piezoelectric patch covering the entire length of the plate has also been considered [7]. The first question addressed in the present paper is the influence of the number and dimensions of the piezoelectric patches on the harvesting efficiency. Existing studies on the damping of vibrations with piezoelectric patches connected to passive circuits considered the influence of a resonant circuit [8]. This motivates the second question in the present paper: what is the influence of a resonant circuit on the non-linear dynamics of the system, and the resulting harvesting efficiency?

The paper is organized as follows. In section II, we present a non linear dynamic model of a plate in an axial flow coupled to electrical circuits trough piezoelectric patches. Continuous and discrete configurations are presented. The numerical methods are also briefly described. Next, a parametric study of the influence on the number and geometries of the piezoelectric patches is presented. In this section, some experimental results are also compared to numerical results. Finally, a study of the influence of a resonant circuit on the energy harvesting efficiency is presented. 


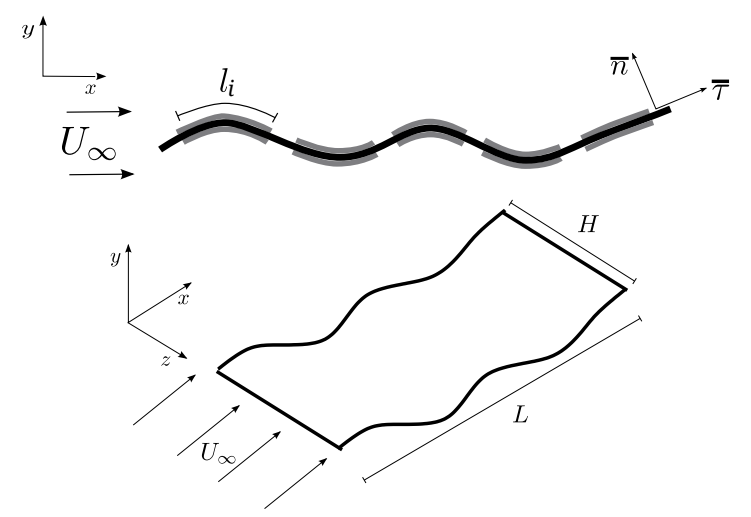

Figure 1: Top: two dimensional flapping of a flexible plate covered by pires of piezoeletric patches. Bottom: flexible plate flapping in uniform axial flow.

\section{II - MODELING}

\section{Euler-Bernoulli beam in an axial flow coupled to electrical circuity via piezoelectricity}

Consider an elastic flexible plate of length $L$, width $H$ and lineic density $\mu$ immersed in an axial flow of uniform velocity $U_{\infty}$, as sketched on figure 1. The fluid's density is $\rho$. The plate is inextensible and clamped at its leading edge. The local orientation of the plate is noted $\theta(S, T)$, where $S$ is the curvilinear coordinate along the streamwise direction and $T$ is time. For simplicity only the $2 \mathrm{D}$ motion of the plate in the $(x y)$ plane is taken into account. Piezoelectric patches of length $l_{p}$, width $H$ and thickness $h_{p}$ are fixed on both sides of the plate. The negative electrodes of the piezo-eletric patches are shunted across the plate while their positive electrodes are connected to an external circuit. The surface density of the charge in each piezo pair is given by [9]:

$$
Q_{i}=\frac{\chi}{l_{i}}[\theta]_{x_{i}^{-}}^{x_{+}^{+}}+c V_{i}
$$

where $l_{i}=x_{i}^{-}-x_{i}^{+}$and $V_{i}$ is the voltage between the positive electrodes of the $i$ th piezo pair whose left and right edges are positioned at $x_{i}^{-}$and $x_{i}^{+}$respectively. $c l_{i}$ is the equivalent capacity of the piezo pair and $\chi$ is the mechanical/piezoelectrical conversion factor of the piezoelectric material. The electrodes are connected to an external circuit. Voltage and charge density are then linked through the following generic relation,

$$
F\left(Q_{i}, V_{i}\right)=0,
$$

whose exact expression depends on the circuits considered.

The voltage $V_{i}$ between both electrodes also generates an internal torque in the piezoeletric patches and thus on the flexible plate. The total internal torque of the bent plate will then be given by $M=B \partial \theta / \partial S-\chi \sum_{i} V_{i} F_{i}$, where $B$ is the flexural rigidity of the plate and piezoeletric patches assembly (see [5]) and $F_{i}$ is the polarization function of the $i$ th patch. In the present approach, $F_{i}=H_{s}\left(x-x_{i}^{-}\right)-H_{s}\left(x-x_{i}^{+}\right)$, with $H_{s}$ the Heaviside step function.

\section{Non-dimensional equations}

To obtain the numerical results presented in the following, non-dimensional equations have been derived with $L$, $L / U_{\infty}, U_{\infty} \sqrt{\mu / c}$ et $U_{\infty} \sqrt{\mu c}$ as characteristic length, time, voltage and charge respectively. Using the conservation of momentum [6], the non-dimensional equation describing the non-linear dynamics of the system are,

$$
\begin{aligned}
\frac{\partial^{2} \bar{x}}{\partial t^{2}} & =\frac{\partial}{\partial s}\left(f_{t} \bar{\tau}-\frac{1}{U^{* 2}} \frac{\partial^{2} \theta}{\partial s^{3}} \bar{n}\right) \\
& +\frac{\alpha}{U^{*}} \sum_{i} v_{i}\left[\delta^{\prime}\left(x_{i}^{-}\right)-\delta^{\prime}\left(x_{i}^{+}\right)\right] \bar{n} \\
& -M^{*} p_{\text {res }}-M^{*} H^{*} m_{a} p_{\text {reac }}, \\
q_{i} & =v_{i}+\frac{\alpha}{U^{*} \gamma}[\theta]_{x_{i}^{-}}^{x^{+}}, \\
f\left(q_{i}, v_{i}\right) & =0,
\end{aligned}
$$


where $p_{\text {reac }}$ and $p_{\text {resis }}$ are respectively the reactive and resistive forces exerted by the flow on the plate and $m_{a}$ is the non-dimensional added mass coefficient of the local cross-section, namely $m_{a}=\pi / 4$ for a flat plate. They have been introduced in the context of slender fish locomotion $[10,11,12]$,

$$
\begin{array}{r}
p_{\text {reac }}=\dot{w}-(w u)^{\prime}+\frac{1}{2} w^{2} \kappa \\
p_{\text {resis }}=\frac{1}{2} C_{d}|w| w,
\end{array}
$$

where $w$ and $u$ are respectively the normal and longitudinal non dimensional components of the plate's velocity relative to the fluid flow.

The set of equations (3-5) depends on the following non-dimensional parameters,

$$
U^{*}=L U_{\infty} \sqrt{\frac{\mu}{B}}, M^{*}=\frac{\rho H L}{\mu}, H^{*}=\frac{H}{L}, \alpha=\frac{\chi}{\sqrt{B c}},
$$

which are respectively the dimensionless flow velocity, mass ratio, aspect ration and piezoelectric coupling factor. In the following, two kinds of electrical circuits will be considered. The first one consists in a shunted resistance of conductance per unit length $g$. In this case, equation (5) becomes,

$$
\beta \dot{q}_{i}+v_{i}=0
$$

The second circuit consists in a resistance and an inductance in parallel. The lineic admittance of the inductance is $1 / \mathcal{L}$. The dynamic equation modeling the circuit is here,

$$
\beta \ddot{q}_{i}+\dot{v}_{i}+\beta \omega_{0}^{2} v_{i}=0
$$

The two additional electrical parameters that are then introduced in the model are,

$$
\beta=\frac{U_{\infty} c}{L g}, \omega_{0}=\frac{L}{U_{\infty} \sqrt{c \mathcal{L}}}
$$

Finally, a plate clamped upstream and free downstream is considered.

\section{Discrete and continuous distributions of electrodes}

The model described above consider a discrete distributions of piezoelectric electrodes, as well as a finite number of corresponding circuits. If the length of the electrodes is small compared to the typical lengthscale of the plate's deformation, it is possible to consider the limit of electrodes of infinitesimal length, as considered in refs [5, 6]. Charge density $q$ and voltage $v$ are now condinuous functions of space and non-dimensional equations read,

$$
\begin{aligned}
\frac{\partial \bar{x}}{\partial t} & =\frac{\partial}{\partial s}\left(f_{t} \bar{\tau}-\frac{1}{U^{* 2}} \frac{\partial^{2} \theta}{\partial s^{2}} \bar{n}+\frac{\alpha}{U^{*}} \frac{\partial v}{\partial s} \bar{n}\right) \\
& -M^{*} p_{r e s}-M^{*} H^{*} m_{a} p_{\text {reac }}, \\
q & =v+\frac{\alpha}{U^{*} \gamma} \theta^{\prime}, \\
f(q, v) & =0 .
\end{aligned}
$$

Equations (9) and (10) remain unchanged, with the indices $i$ ommited.

\section{Efficiency of the system}

The definition of the efficiency used in the present article is similar to that classically used in windmill studies. It is defined as the ratio between the mean harvested power and the kinetic energy flux through the surface occupied by the energy harvesting system,

$$
\eta=\frac{\mathcal{P}_{e}}{\mathcal{P}_{f}}
$$

In the present approach, the harvested power is that dissipated in the resistance. The definition of the surface occupied by the harvesting system is less trivial in the present case than that of windmill, where this surface is the disc occupied by the rotating windmill. Here, we have chosen the rectangle defined by the width of the plate and its maximum displacement during the oscillation. 


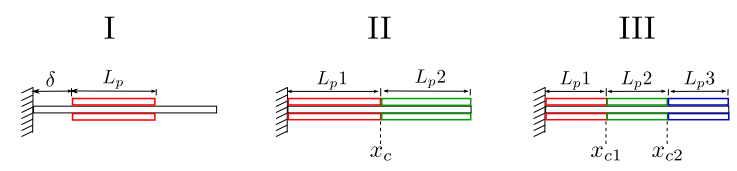

Figure 2: Schematic view and notations in the three studied cases: one, two or three electrodes.

\section{Numerical methods}

Following [13], equation (5) is projected onto $x$ and $y$ in order to obtain two equations for $x(s, t)$ and $y(s, t)$ respectively. The $\mathrm{x}$ projection is used to eliminate the tension term from the $y$ projection. Finally, $x$ and its derivatives are eliminated using the inextensibility condition.

Two kinds of numerical approaches have then been used to obtain the results presented in the following. In the case of a discrete system, the equations for $y(s, t)$ are first written in a weakly non-linear form by keeping only terms up to $\mathcal{O}\left(y^{3}\right)$, as done in [13] in the uncoupled case. A Galerkin discretization is next used: the displacement $y$ is written as a sum of $N$ clamped-free beam modes and the equilibrium equations are projected on these beam modes. One obtain a set of $N+N_{p}$ ordinary differential equations which are integrated using an implicit numerical scheme.

In the case of a continuous system, nonlinear equations (12-14) are solved using an implicit second order timestepping scheme [14]. The flag is meshed by Chebyshev-Lobatto nodes, and a Chebyshev collocation method is used to compute spatial derivatives and integration. At each time step, the resulting nonlinear system is solved using Broyden's method [15]. The simulation is started with a perturbation in the flag's orientation $(\theta(s, t=0) \neq 0)$, and is carried out over a sufficiently long time frame so as to reach a permanent regime. The quantities necessary for evaluating the efficiency are then obtained as follows: the harvested energy is computed as the time average of the dissipation in the resistive elements, and the flapping amplitude is defined as the root-mean-square amplitude of the trailing edge, both within the permanent regime.

\section{III - RESULTS}

In this section, different parametric studies of the efficiency of the system are presented. We focus only on the postcritical dynamics of the system, once flutter instability has appeared. Only a few representative sets of mechanical parameters $M^{*}$ and $U^{*}$ are investigated. The coupling coefficient is fixed at 0.3 , which corresponds to reasonable values one could obtain using PZT patches on steel plates [5].

At first we show the number and geometry of the electrodes affect the efficiency of the system. The cases of 1,2 and 3 electrodes are successively considered. Next, we present a numerical/experimental compraison of the efficiency for a piezoelectric patch of variable length. The circuit is a purely resitive circuit. Finally a case of a continuous distribution is considered, where the harvesting circuit also contains an inductance.

\section{Influence of the number and distribution of electrodes}

Case I: one electrode We consider here that one piezoelectric patch of length $l_{p}$ covers partially the surface of the plate. It is fixed at a distance $\delta$ from the clamped end. In the present approach, it is supposed that the present piezoelectric patch does not affect the density and bending rigidity of the sandwich plate. For a study that takes into account this effect, one could refer for instance to reference [16], in the context of the passive damping of vibrating beams.

Figure 3 presents the efficiency of the harvesting system in the $\left(\delta, l_{p}\right)$ plane, for two values of the mass ratio $M^{*}$, just above the instability threshold. Each colored rectangle of these plots corresponds to one numerical simulation where the value of $\beta$ was adjusted to reach maximum efficiency.

For $M^{*}=0.6$, the maximum efficiency is achieved for $\delta \sim 0.4$ and $l_{p} \sim 0.5$. In this case, the electrode resides mainly at the second half of the clamped-free plate. This result differ significantly to that obtained in works on energy harvesting from vibrating beams, where the piezoelectric patch is generally placed at the clamp or covering the whole length $[17,7]$.

For $M^{*}=10$, three local maxima of the efficiency in the $\left(\delta, l_{p}\right)$ plane can be observed. They correspond to an electrode of length $l_{p} \sim 0.3$ at $\delta \sim 0.3,0.5$ and 0.75 respectively. The presence of more local maxima with shorter electrodes is explained by the fact that the higher is $M^{*}$, the higher is the number of spatial oscillation is the unstable mode [18].

Case II: two electrodes The case with two electrodes covering the whole plate is now considered. The parametric study now depends on one parameter only: the length of the first electrode, noted $x_{c}$. On figure 4 , the parametric 

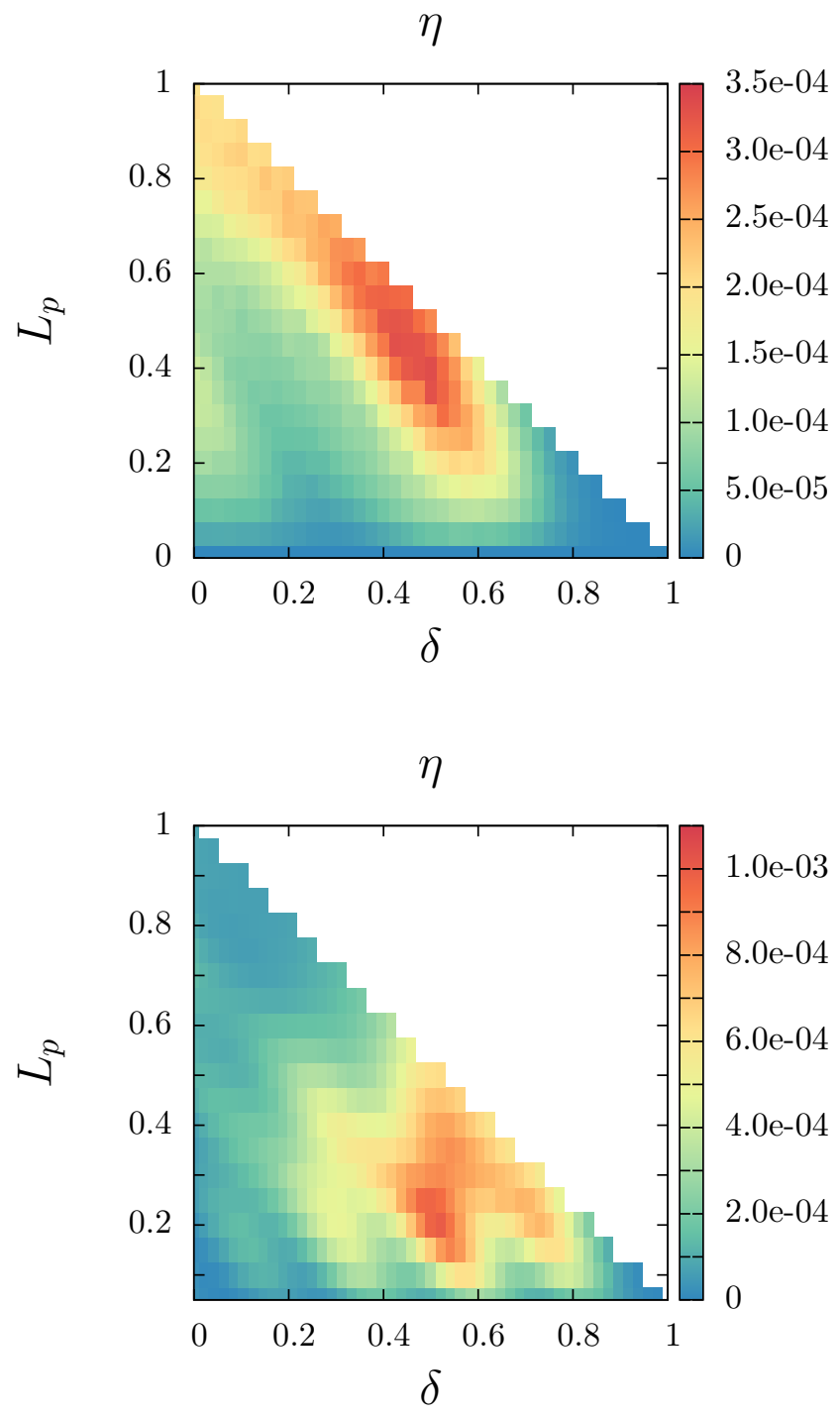

Figure 3: Case of one electrode per side of the flag. Effect of the electrode's position and length on the energy harvesting efficiency, at $U^{*}=13, M^{*}=0.6$ (top) and $M^{*}=10$ (bottom). For each pair of geometrical parameters, $\beta$ is varied to reach the maximum efficiency.

study is done in the same two cases: $M^{*}=0.6$ and $M^{*}=10$, with $U^{*}$ chosen just above the instability threshold, $U^{*}=13$ and 8.5 respectively. For $M^{*}=0.6$, the maximum value of $\eta$ is found for $x_{c} \sim 0.35$ and is approximately twice the minimum efficiency $\eta$. For $M^{*}=10$ we find two local maxima, around $x_{c}=0.5$ and $x_{c}=0.7$ respectively. These maxima represent a significant improvement of the efficiency. Indeed, in this case, the maximum efficiency is approximately 9 times larger than the minimum efficiency. The contribution of each piezoelectric pair on the efficiency is also plotted on this figures, showing that when the optimal efficiency is reached, the contribution of the downstream piezoelectric pair is significantly higher than the upstream one. This is consistent with the results of case I presented above.

Case III: three electrodes The case with three electrodes covering the entire plate is now adressed in the same two configurations as before. There are now two parameters, $x_{c 1}$ and $x_{c 2}$, which are the junctions between two piezoelectric patches.

For $M^{*}=0.6$ the maximum efficiency is reached for $x_{c 1} \sim 0.4$ and $x_{c 2} \sim 0.9$. In this configuration, the second piezoelectric patch is approximately the same as in the one of case $\mathrm{I}$, the two other patches covering the remaining space on the plate. For $M^{*}=10$, the point of maximum efficiency $\left(x_{c 1}=0.5, x_{c 2}=0.7\right)$ can not be related to the optimal configuration of case I. For this large value of the mass ratio, the sensibility of the efficiency with the parameters is important and it will be consequently more difficult to reach the optimal efficiency in practical applications. 

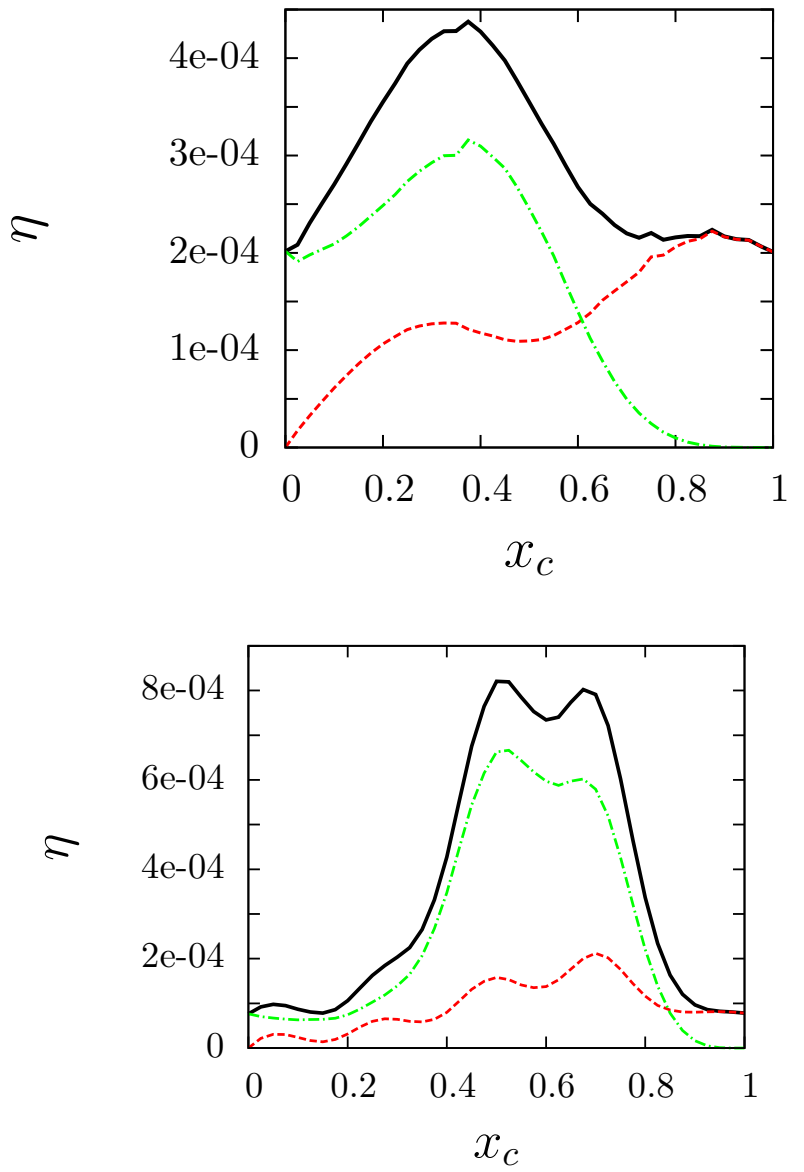

Figure 4: Case of two electrodes per side of the flag. Effect of the position of the cut between the two electrodes on the energy harvesting efficiency, at $U^{*}=13, M^{*}=0.6$ (top) and $M^{*}=10, U^{*}=8.5$ (bottom). For each value of $M^{*}$, $\beta$ is varied to reach the maximum efficiency. Solid black line shows the total efficiency of the configuration. Red and green lines show the individual contribution of each electrode. 

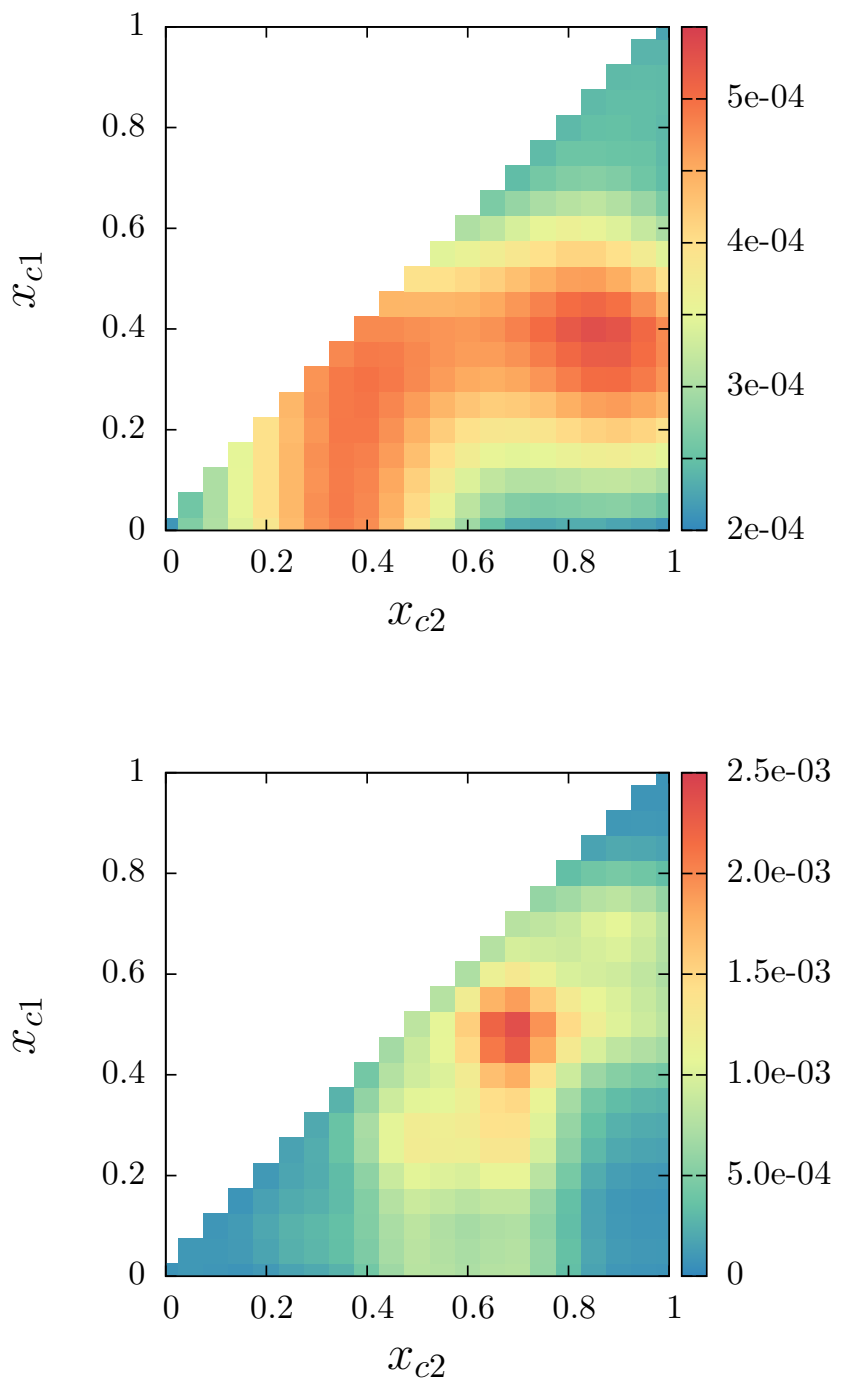

Figure 5: Case of three electrodes per side of the flag. Effect of the position of the cut between two consecutive electrodes on the energy harvesting efficiency, at $U^{*}=13, M^{*}=0.6$ (top) and $M^{*}=10$ (bottom). For each pair of $x_{c 1}$ and $x_{c 2}, \beta$ is varied to reach the maximum efficiency. 


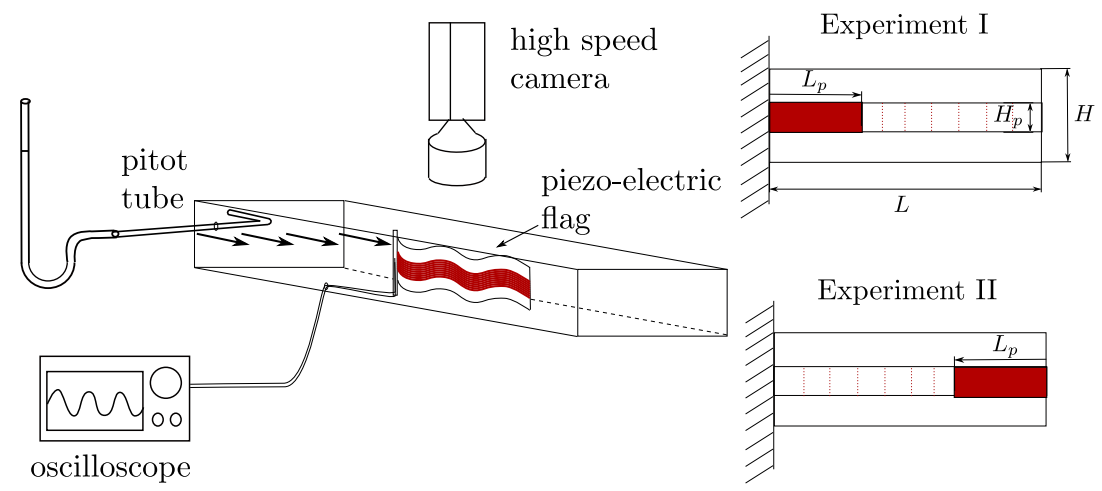

Figure 6: Experimental setup and description of the two tested configurations.

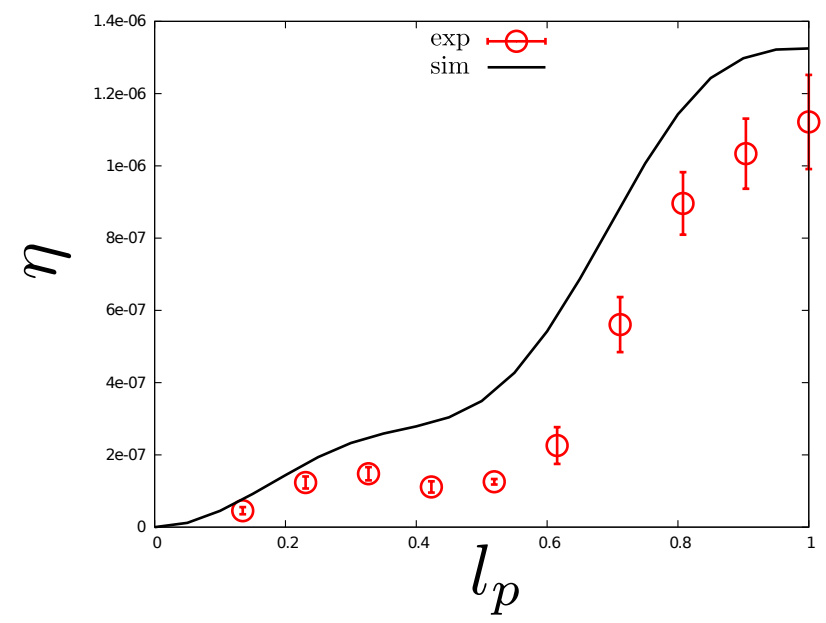

Figure 7: Numerical and experimental energy harvesting efficiency $\eta$ as function of the dimensionless length of the piezoelectric patch $l_{p}$ with $\delta=0, U^{*}=14.6, M *=0.68, H^{*}=0.3, \alpha=.073$. The shunting resistance is adjusted so get maximum dissipated power.

Before the presentation of some experimental results, let us discuss briefly the main results of this section. First of all, we have presented results obtained by a weakly nonlinear model discretized using a small set of Galerkin modes, allowing fast computations. Considering one, two or three electrodes covering entierely or partially the plate, we have revealed the geometrical distributions that maximize the efficiency. We show that the optimal distributions strongly depend on the mass ratio $M^{*}$. In the single-electrode case, we also showed that letting an extremity of the electrode be at the clamp is far from being the optimal configuration. It is indeed possible to increase the efficiency by a factor 2 at $M^{*}=0.6$ and 10 at $M^{*}=10$ when looking for the best position and length of the electrode.

\section{Experiments}

A series of experimental measurements is now presented. A schematic view of the experimental setup is given in Fig. 6. Two experiments have been conducted, which consist basically in considering the case I, with one extremity of the electrode fixed at $x=0$ or $x=1$. The former (experiment I on Fig. 6) is achieved by fixing $\delta=0$ and varying $l_{p}$ from 0 to 1 . The latter (experiment II on Fig. 6 ) is achieved by varying $\delta$ and imposing $l_{p}=1-\delta$. In both experiments the studied plates are composed of a mylar sheet of length $12 \mathrm{~cm}$, width $2.5 \mathrm{~cm}$ and thickness $100 \mu \mathrm{m}$. A piezoelectric PVDF film fixed on each side of the sheet. The width of the piezoelectric sheet in the spanwise direction is smaller than the width of the plate, in order to reduce the influence of $\delta$ and $l_{p}$ on the local flexural rigidity and lineic mass of the system. PVDF films are covered with an external $\mathrm{Cr} / \mathrm{Au}$ which serves as the electrode. During the experiments the $\mathrm{Cr} / \mathrm{Au}$ layer is sectioned in order to adjust the desired value of $\delta$ and $l_{p}$. The thickness of the PVDF film in experiment 1 is $40 \mu \mathrm{m}$ while in experiment 2 is $50 \mu \mathrm{m}$. The system is placed in a wind tunnel of rectangular transversal section $10 \mathrm{~cm}$ wide and $5 \mathrm{~cm}$ high with transparent walls that allow taking images from the outside. At a given velocity above the instability threshold, the voltage is measured across the electrodes of the piezoelectric patches with a variable resistance adjusted so that the dissipated power is maximum. The efficiency is then calculated using equation (15).

On figure 7 , the efficiency is plotted against $l_{p}$ for $\delta=0$. Numerical and experimental results are plotted on the 


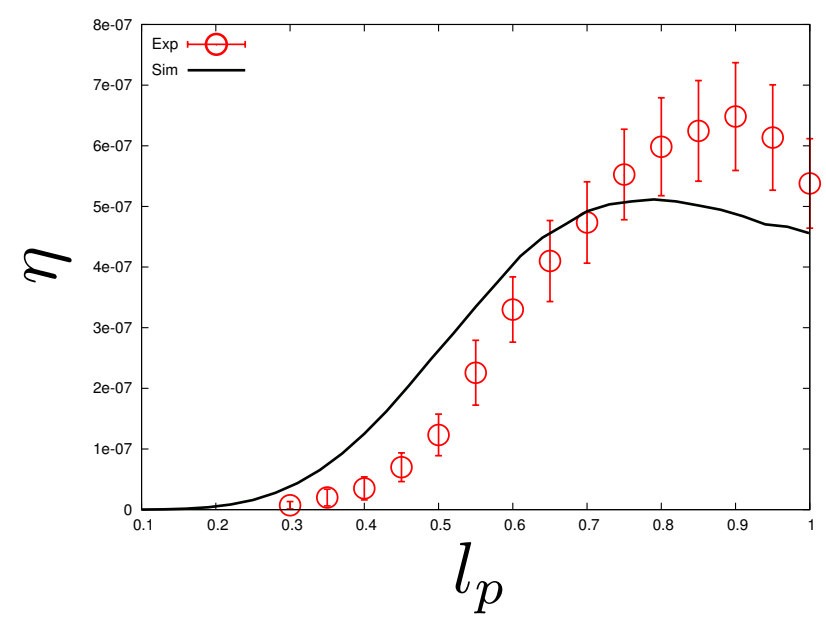

Figure 8: Numerical and experimental energy harvesting efficiency $\eta$ as function of the dimensionless length of the piezoelectric patch $l_{p}$ with $\delta=1-l_{p}, U^{*}=15.7, M^{*}=0.61, H^{*}=0.3, \alpha=0.062$. The shunting resistance is adjusted so get maximum dissipated power.
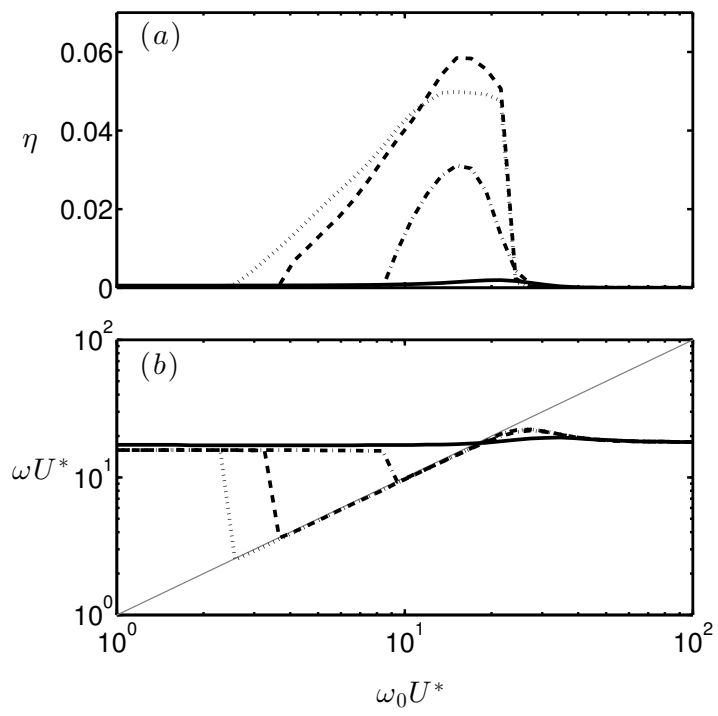

Figure 9: Efficiency $\eta$ (top) and flapping frequency $U^{*} \omega$ (bottom) as function of $U^{*} \omega_{0}$ for $\beta=0.05$ (solid), $\beta=1$ (dash-dotted), $\beta=4$ (dashed) and $\beta=8$ (dotted). For all figures, $\alpha=0.3, M^{*}=1$ and $U^{*}=13$.

same figure, and show a good agreement. The maximum efficiency is achieved when the piezoelectric patch occupy the whole length of the plate.

On figure 8 , the efficiency is plotted against $l_{p}$ with $l_{p}=1-\delta$, so that the piezoelectric patch lies between $x=\delta$ and $x=1$. Here again, there is a good agreement between numerical results and experiments. The maximum efficiency is reached when the piezoelectric patch upstream extremity is a $x \sim 0.1$.

Hence, there is an overall good agreement between theory and experiments with respect to the influence of the geometry of the electrode on the efficiency of the harvester. The slight discrepancies might be due difficulties of controlling the parameters during the manufacturing process and to the influence of the piezoelectric patch on the local bending rigidity and density, which is not taken into account bythe model.

\section{IMPROVEMENT OF EFFICIENCY BY A LOCK-IN MECANISM}

In this section, a different harvesting circuit is considered. It now consists in a resistance and an inductance in parallel. Due to the fact that the piezoelectric patch behaves like a capacitor, the resulting circuit consists in an RLC circuit that can enter in resonnance with the forcing coming from the piezoelectric coupling. In the present case, the continuous limit is considered and numerical results using the full non-linear model are presented.

On figure $9(\mathrm{a})$, the efficiency is plotted against $\omega_{0}$ for different values of $\beta$. The other parameters are fixed to 

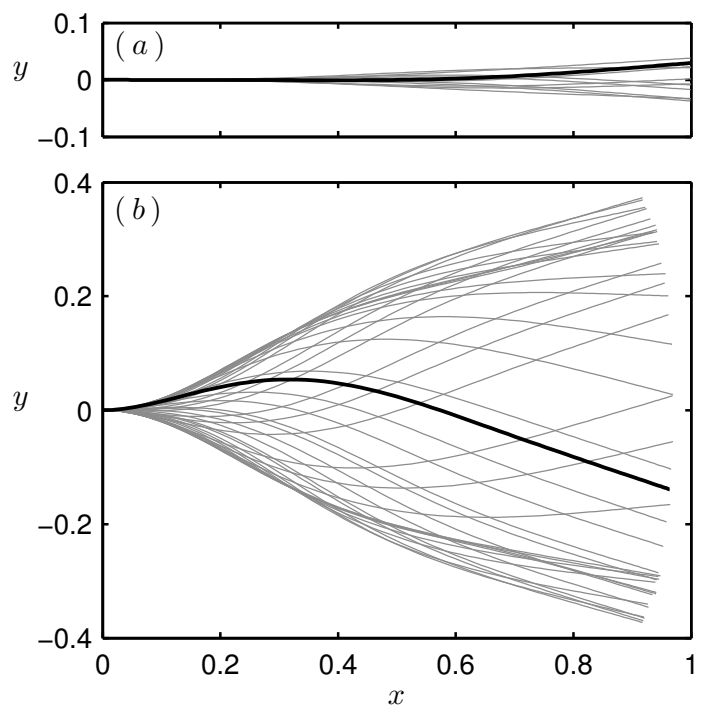

Figure 10: Flag's deformation during one flapping cycle, comparison between no lock-in (a) and lock-in (b) cases. For all figures, $\alpha=0.3, M^{*}=1$ and $U^{*}=13$.

$M^{*}=1, U^{*}=13, H^{*}=0.5$ and $\alpha=0.3$. In a range of $\omega_{0}$ around unity, the efficiency reach a maximum of the order of $10^{-2}$, which is significantly higher than what can be achieved with purely resistive circuits. The flapping frequency is plotted on figure 9 and shows that when high efficiencies are obtained, the flapping frequency $\omega=\omega_{0}$. This phenomenon is referred to as a frequency lock-in, and is mainly reported in works on Vortex-Induced Vibration (VIV), [19]. In this phenomenon, the frequency of an active oscillator (the flapping flag) is dictated by the natural frequency of a coupled passive oscillator (the circuit).

The deformation of the flag during one period of oscillation is plotted on figure 10 in a case without lock-in $\left(\omega_{0}=3.25\right)$, and in a case with lock-in $\left(\omega_{0}=4.12\right)$. This plot shows that when the lock-in occurs, the fluttering amplitude of the flag is significantly larger. This emphasizes that the efficiency is increased not only because of a resonance in the $R L C$ circuit, but also because of an increase of the flapping amplitude.

\section{CONCLUSION}

In this article a model of a piezoelectric flag coupled with energy harvesting circuits has been presented. The discrete and continuous configurations have been defined. When the number of piezoelectric patches tends to infinity and their length is small compared to the typical deformations of the flag, a continuous electrical model can be used.

In all the paper, we focused on the energy harvesting efficiency of the system, whose definition is similar to that used in windmill studies. First, the influence of the number and arrangement of the piezoelectric patches has been characterized. Optimal positions have been found, which depends on the mass ratio $M^{*}$. As in previous studies $[5,6]$, higher efficiencies are obtained at higher mass ratios, but then the sensibility to the geometrical parameters is larger. Finally, a lock-in phenomenon has been evidenced. The latter appears when a resonant circuit is considered and corresponds to a situation where the flapping frequency is dictated by the frequency of the electrical circuit. In the theoretical configuration presented above, this phenomenon is able to significantly increase the efficiency of the harvester. Experiments should now be performed to assess the effectiveness of the efficiency increase after introducing inductive elements to the energy harvesting circuits.

\section{acknowledgment}

This work received financial support from the "Laboratoire d'Excellence" LASIPS ("PIEZOFLAG" project), This work was supported by the "Laboratoire d'Excellence" LASIPS ("PIEZOFLAG" project) and by the French National Research Agency ANR (Grant ANR-2012-JS09-0017). S. M. also acknowledges the support of a Marie Curie International Reintegration Grant within the 7th European Community Framework Programme (PIRG08-GA-2010- 276762). 


\section{References}

[1] Westwood, A., 2004. "Ocean power: Wave and tidal energy review". Refocus, 5(5), pp. 50-55.

[2] Elvin, N., and Erturk, A., 2013. Advances in energy harvesting methods. Springer.

[3] Allen, J., and Smits, A., 2001. "Energy harvesting eel". Journal of fluids and structures, 15, pp. 629-640.

[4] Taylor, G., Burns, J., S.M., K., W.B., P., and Welsh, T., 2001. "The Energy Harvesting Eel: A Small Subsurface Ocean/River Power Generator". IEEE Journal of Oceanic Engineering, 26(4), pp. 539-547.

[5] Doaré, O., and Michelin, S., 2011. "Piezoelectric coupling in energy-harvesting fluttering flexible plates: linear stability analysis and conversion efficiency". Journal of Fluids and Structures, 27(8), pp. 1357-1375.

[6] Michelin, S., and Doaré, O., 2013. "Energy harvesting efficiency of piezoelectric flags in axial flows". Journal of Fluid Mechanics, 714, pp. 489-504.

[7] Akcabay, D. T., and Young, Y. L., 2012. "Hydroelastic response and energy harvesting potential of flexible piezoelectric beams in viscous flow". Physics of Fluids, 24(5), p. 054106.

[8] Hagood, N., and von Flotow, A., 1991. "Damping of structural vibrations with piezoelectric materials and passive electrical networks". Journal of Sound and Vibration, 146(2), pp. 243-268.

[9] Preumont, A., 2002. Vibration Control of Active Structures: An Introduction, 2nd ed. Kluwer Academic Plublishers.

[10] Taylor, G., 1952. "Analysis of the Swimming of Long and Narrow Animals". Proceedings of the Royal Society of London. Series A. Mathematical and Physical Sciences, 214(1117), pp. 158-183.

[11] Lighthill, M. J., 1971. "Large-amplitude elongated-body theory of fish locomotion". Proceedings of the Royal Society of London. Series B. Biological Sciences, 179(1055), pp. 125-138.

[12] Candelier, F., Boyer, F., and Leroyer, A., 2011. "Three-dimensional extension of Lighthill's large-amplitude elongated-body theory of fish locomotion". Journal of Fluid Mechanics, 674, p. 196.

[13] Eloy, C., Kofman, N., and Schouveiler, L., 2012. "The origin of hysteresis in the flag instability". Journal of Fluid Mechanics, 691, pp. 583-593.

[14] Alben, S., 2009. "Simulating the dynamics of flexible bodies and vortex sheets". Journal of Computational Physics, 228(7), pp. 2587-2603.

[15] Broyden, C. G., 1965. "A class of methods for solving nonlinear simultaneous equations". Mathematics of

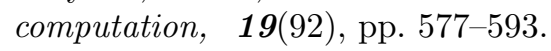

[16] Thomas, O., Deue, J. F., and Ducarne, J., 2009. "Vibrations of an elastic structure with shunted piezoelectric patches: efficient finite element formulation and electromechanical coupling coefficients". International Journal for Numerical Methods in Engineering, $80(2)$, OCT 8, pp. 235-268.

[17] Dunnmon, J. A., Stanton, S. C., Mann, B. P., and Dowell, E. H., 2011. "Power extraction from aeroelastic limit cycle oscillations". Journal of Fluids and Structures, 27(8), pp. 1182-1198.

[18] Eloy, C., Lagrange, R., Souilliez, C., and Schouveiler, L., 2008. "Aeroelastic instability of cantilevered flexible plates in uniform flow". Journal of Fluid Mechanics, 611, pp. 97-106.

[19] Williamson, C. H. K., and Govardhan, R., 2004. "Vortex-induced vibrations". Annu. Rev. Fluid Mech., 36, pp. $413-455$. 